\title{
Disturbance Due to Thermomechanical Sources in Porothermoelastic Medium
}

\author{
R. Kumar ${ }^{\mathrm{a}, 1}$ and I. A. Abbas ${ }^{\mathrm{b}, \mathrm{c}, 2}$ \\ a Department of Mathematics, Kurukshetra University, Kurukshetra, Haryana, India \\ b Department of Mathematics, Faculty of Science and Arts-Khulais, King Abdulaziz University, \\ Jeddah, Saudi Arabia \\ ${ }^{c}$ Department of Mathematics, Faculty of Science, Sahag University, Sohag, Egypt \\ ${ }^{1}$ rajneesh_kuk@rediffmail.com \\ 2 ibrabbas7@yahoo.com
}

УДК 539.4

\section{Возмущения от термомеханических источников в пористой термоупругой среде}

\author{
Р. Кумар ${ }^{\text {a }, ~ И . ~ А . ~ А б б а с ~}{ }^{б, \mathrm{~B}}$ \\ a Отделение математики университета Курукшетра, Харьяна, Индия \\ ${ }^{\sigma}$ Отделение математики университета Кинг Абдулазиз, Джедда, Саудовская Аравия

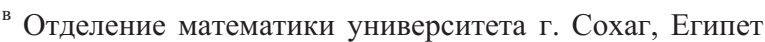

Исследована двухмерная задача о возникновении возмущений от механических и термических источников в однородной теплопроводящей пористо-упругой среде. Для решения этой задачи использованы преобразования Лапласа и Фурье. Для демонстрации перспективности данного подхода рассмотрены различные случаи с точечными и распределенными источниками. С помощью методики численной инверсии выполнены преобразования компонент перемещений, напряжений, давления в порах и температурных флуктуаций. Влияние пористости на указанные параметры представлено в виде графиков. Рассмотрены некоторые специальнье случаи, имеющие практический интерес.

Ключевые слова: пористая термоупругая среда, преобразование Лапласа, преобразование Фурье, точечный источник, равномерно распределенный источник, линейно распределенный источник.

Introduction. Coupled thermal and poro-mechanical processes play an important role in a number of problems of interest in the geomechanics such as stability of borehole and permeability enhancement in geothermal reservoirs or high temperature petroleum bearing formations. A thermoporoelastic approach combine the theory of heat conduction with poroelastic constitutive equations and coupling the temperature field with the stresses and pore pressure.

Most of the modern engineering structures are generally made up of multiphase porous continuum, the classical theory, which represent a fluid saturated porous medium as a single phase material, is inadequate to represent the mechanical behavior of such material especially when the pore are filled with liquid. In this context the solid and liquid phases have different motions. Due to these different motions, the different material properties and the complicated geometry of pore structures, the mechanical behavior of a fluid saturated porous medium is very complex and difficult. 
Based on the work of von Terzaghi [1, 2], Biot [3] proposed a general theory of threedimensional deformations of liquid saturated porous solid. The Biot theory is based on the assumption of compressible constituents and till recently, some of his results have taken as standard references and basis for subsequent analysis in acoustic, geophysics, and other such fields [4-6].

Kumar and Hundal [7] discussed the wave propagation in a fluid saturated incompressible porous medium. Bai and $\mathrm{Li}$ [8] found a solution for cylindrical cavity in saturated thermoporoelastic medium by using Laplace transform and numerical Laplace transform inversion. Bai [9-11] discussed different thermal source problem in a saturated porous media.

Kaushal et al. [12] studied the response of frequency domain in generalized thermoelasticity with two temperature. Jabbari and Dehbani $[13,14]$ studied axisymmetric and spherical symmetric problems in porothermoelastic solids. Abbas [15] studied the natural frequencies of a poroelastic hollow cylinder. The counterparts of our problem in the contexts of the thermoelasticity theories have been considered by using analytical and numerical methods [16-29].

In the present paper, we obtain the components of displacement, stress, pore pressure and temperature change due to concentrated source and distributed source in thermoporoelastic medium. Laplace and Fourier transforms are used to investigate the problem. Numerical inversion technique is applied to obtain the resulting quantities in a physical domain. The resulting quantities are shown graphically to depict the effect of porosity.

Governing Equations. Following Jabbari and Dehbani [30], the field equations are

$$
\begin{gathered}
(\lambda+\mu) \nabla \nabla \vec{u}+\mu \nabla^{2} \vec{u}-\alpha \nabla p-\beta \nabla T=\rho \frac{\partial^{2} \vec{u}}{\partial t^{2}}, \\
\frac{k}{\gamma_{w}} \nabla^{2} p-\alpha_{p} \dot{p}-Y \dot{T}-\alpha \operatorname{div} \dot{\vec{u}}=0, \\
K \nabla^{2} T-Z T_{0} \dot{T}+Y T_{0} \dot{p}-\beta T_{0} \operatorname{div} \dot{\vec{u}}=0, \\
\sigma_{i j}=\lambda u_{k, k} \delta_{i j}+\mu\left(u_{i, j}+u_{j, i}\right)-\alpha p \delta_{i j}-\beta T \delta_{i j},
\end{gathered}
$$

where $\vec{u}$ is the displacement component, $p$ is the pore pressure, $\rho$ is the bulk mass density, $\alpha=1-C_{s} / C$ is the Biot coefficient, $C_{s}=3\left(1-2 v_{s}\right) / E_{s}$ is the coefficient of volumetric compression of solid grain, with $E_{s}$ and $v_{s}$ being the elastic modulus and Poisson's ratio of solid grain, $C=3(1-2 v) / E$ is the coefficient of volumetric compression of solid skeleton, with $E$ and $v$ being the elastic modulus and Poisson's ratio of solid skeleton, $T_{0}$ is initial reference temperature, $\beta=3 \alpha_{s} / C$ is the thermal expansion factor, $\alpha_{s}$ is the coefficient of linear thermal expansion of solid grain, $Y=3\left(n \alpha_{w}+(\alpha-n) \alpha_{s}\right)$ and $\alpha_{p}=n\left(C_{w}-C_{s}\right)+\alpha C_{s}$ are coupling parameters, $\alpha_{w}$ and $C_{w}$ are the coefficients of linear thermal expansion and volumetric compression of pure water, $n$ is the porosity, $k$ is the hydraulic conductivity, $\gamma_{w}$ is the unit of pore water, $Z=\frac{(1-n) \rho_{s} c_{s}+n \rho_{w} c_{w}}{T_{0}}-3 \beta \alpha_{s}$ is coupling parameter, $\rho_{w}$ and $\rho_{s}$ are densities of pore water and solid grain, $c_{w}$ and $c_{s}$ are heat capacities of pore water and solid grain, and $K$ is the coefficient of heat conductivity.

Formulation of the Problem. We consider homogeneous porothermoelastic half space $x_{3} \geq 0$ of a rectangular Cartesian coordinate system $\left(x_{1}, x_{2}, x_{3}\right)$ having origin at the surface $x_{3}=0, x_{3}$ is the axis pointing vertically downward in the medium. A mechanical 


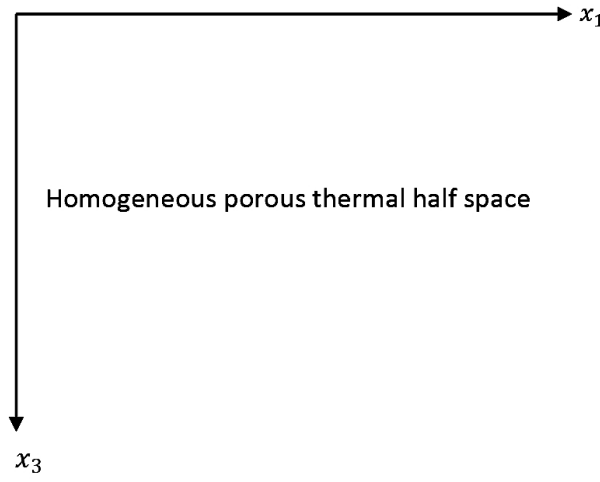

Fig. 1. Geometry of the problem.

or thermal source is assumed to be acting at the origin. We restrict our analysis to the plane strain parallel to $x_{1}-x_{3}$ plane. The complete geometry of the problem is shown in Fig. 1. For two-dimensional problem we take the displacement vector $\vec{u}$ as

$$
\vec{u}=\left(u\left(x_{1}, x_{3}\right), 0, w\left(x_{1}, x_{3}\right)\right) .
$$

To facilitate the solution, the following dimensionless quantities are introduced as

$$
\begin{aligned}
& x_{1}^{\prime}=\frac{\omega^{*}}{c_{1}} x_{1}, \quad x_{3}^{\prime}=\frac{\omega^{*}}{c_{1}} x_{3}, \quad u_{1}^{\prime}=\frac{\omega^{*} \rho c_{1}}{\beta T_{0}} u_{1}, \quad u_{3}^{\prime}=\frac{\omega^{*} \rho c_{1}}{\beta T_{0}} u_{3}, \quad p^{\prime}=\frac{p}{\beta T_{0}}, \\
& c_{1}^{2}=\frac{\lambda+2 \mu}{\rho}, \quad t^{\prime}=\omega^{*} t, \quad T^{\prime}=\frac{T}{T_{0}}, \quad \omega^{*}=\frac{Z T_{0} c_{1}^{2}}{K}, \quad \sigma_{33}^{\prime}=\frac{\sigma_{33}}{\beta T_{0}}, \quad \sigma_{31}^{\prime}=\frac{\sigma_{31}}{\beta T_{0}} .
\end{aligned}
$$

The expressions relating the displacement components $u_{1}\left(x_{1}, x_{3}, t\right)$ and $u_{3}\left(x_{1}, x_{3}, t\right)$ to the scalar potentials $\Phi$ and $\Psi$ in dimensionless form are given by

$$
u_{1}=\frac{\partial \Phi}{\partial x_{1}}-\frac{\partial \Psi}{\partial x_{3}}, \quad u_{3}=\frac{\partial \Phi}{\partial x_{3}}+\frac{\partial \Psi}{\partial x_{1}} .
$$

We define the Laplace and Fourier transforms as follows:

$$
\begin{gathered}
\bar{f}(s)=\int_{0}^{\infty} f(t) e^{-s t} d t, \\
\widetilde{f}(\xi)=\int_{-\infty}^{\infty} \bar{f}(\xi) e^{i \xi x_{1}} d x .
\end{gathered}
$$

Applying Laplace and Fourier transforms defined by (8) and (9) on Eqs. (1)-(3) and with the aid of (5)-(7) and after some simplifications we obtain

$$
\begin{gathered}
{\left[A_{1} \frac{d^{6}}{d x_{3}^{6}}+A_{2} \frac{d^{4}}{d x_{3}^{4}}+A_{3} \frac{d^{2}}{d x_{3}^{2}}+A_{4}\right] \widetilde{\bar{\Phi}}=0,} \\
a_{1}\left(\frac{d^{2}}{d x_{3}^{2}}-\xi^{2}\right) \widetilde{\Psi}-a_{4} s^{2} \tilde{\bar{\Psi}}=0,
\end{gathered}
$$


where

$$
\begin{gathered}
A_{1}=b_{1} b_{4}\left(1+a_{1}\right), \\
A_{2}=3 \xi^{2} b_{1} b_{4}\left(1+a_{1}\right)-s\left(1+a_{1}\right)\left(b_{1} b_{5}+b_{2} b_{4}\right)-a_{4} b_{1} b_{4} s^{2}-a_{2} b_{4} s-a_{3} b_{1} s, \\
A_{3}=3 \xi^{4} b_{1} b_{4}\left(1+a_{1}\right)+2 \xi^{2} s\left(1+a_{1}\right)\left(b_{1} b_{5}+b_{2} b_{4}\right)+s^{2}\left(1+a_{1}\right)\left(b_{1} b_{5}+b_{2} b_{4}\right)+ \\
+2 \xi^{2} s^{2} a_{4} b_{1} b_{4}+a_{4} s^{3}\left(b_{1} b_{5}+b_{2} b_{4}\right)+2 \xi^{2} a_{2} b_{4} s-a_{2} s^{2}\left(b_{3}-b_{5}\right)+ \\
+2 \xi^{2} a_{3} b_{1} s+a_{3} s^{2}\left(b_{6}+b_{2}\right), \\
A_{4}=-\xi^{6} b_{1} b_{4}\left(1+a_{1}\right)-s^{4}\left(1+a_{1}\right)\left(b_{1} b_{5}+b_{2} b_{4}\right)+s^{2} \xi^{2}\left(1+a_{1}\right)\left(b_{1} b_{5}+b_{2} b_{4}\right)- \\
-a_{4} b_{1} b_{4} s^{2} \xi^{4}-a_{4} s^{3} \xi^{2}\left(b_{1} b_{5}+b_{2} b_{4}\right)-a_{4} s^{4}\left(b_{2} b_{5}+b_{3} b_{6}\right)-a_{2} b_{4} s \xi^{4}+ \\
\quad+a_{2} s^{2}\left(b_{3}-b_{5}\right) \xi^{2}-\xi^{4} a_{3} b_{1} s-a_{3} s^{2} \xi^{2}\left(b_{6}+b_{2}\right), \\
\mu \\
b_{1}=\frac{\rho \omega^{*} \beta T_{0}}{\gamma_{w} \alpha c_{1}^{2}}, \quad a_{2}^{2}=\frac{\alpha \rho c_{1}^{2}}{\lambda+\mu}, \quad a_{3}=\frac{\rho c_{1}}{(\lambda+\mu)}, \quad a_{4}=\frac{\rho c_{1}^{2}}{\lambda+\mu}, \\
b_{3}=\frac{Y T_{0}}{\alpha}, \quad b_{4}=\frac{K \omega^{*}}{\beta c_{1}^{2}}, \quad b_{5}=\frac{Z T_{0}}{\beta}, \quad b_{6}=Y T_{0} .
\end{gathered}
$$

The solution of Eqs. (10) and (11) satisfying the radiation conditions that $\widetilde{\bar{\Phi}}, \tilde{\bar{\Psi}}, \tilde{\bar{p}}$, and $\widetilde{\bar{T}} \rightarrow 0$ as $x_{3} \rightarrow \infty$ we obtain the value of $\widetilde{\Phi}, \widetilde{\Psi}, \widetilde{\bar{p}}$, and $\widetilde{\bar{T}}$ as

$$
\begin{gathered}
(\tilde{\bar{\Phi}}, \tilde{\bar{p}}, \tilde{\bar{T}})=\sum_{i=1}^{3}\left(1, r_{i}, s_{i}\right) B_{i} e^{-m_{i} x_{3}}, \\
\widetilde{\widetilde{\Psi}}=B_{4} e^{-m_{4} x_{3}}
\end{gathered}
$$

where $m_{1}, m_{2}$, and $m_{3}$ are the roots of Eq. (10), and $m_{4}=\sqrt{A_{5}}, A_{5}=\xi^{2}+\frac{a_{4}}{a_{1}} s^{2}$, and the coupling constants are given by

$$
\begin{gathered}
r_{i}=\frac{b_{4} s\left(m_{i}^{2}-\xi^{2}\right)^{2}+\left(b_{3} s^{2}-b_{5} s^{2}\right)\left(m_{i}^{2}-\xi^{2}\right)}{b_{1} b_{4}\left(m_{i}^{2}-\xi^{2}\right)^{2}-\left(b_{1} b_{5} s+b_{2} b_{4} s\right)\left(m_{i}^{2}-\xi^{2}\right)+\left(b_{2} b_{5} s^{2}+b_{3} b_{6} s^{2}\right)}, \\
s_{i}=\frac{b_{1} s\left(m_{i}^{2}-\xi^{2}\right)^{2}-\left(b_{6} s^{2}+b_{2} s^{2}\right)\left(m_{i}^{2}-\xi^{2}\right)}{b_{1} b_{4}\left(m_{i}^{2}-\xi^{2}\right)^{2}-\left(b_{1} b_{5} s+b_{2} b_{4} s\right)\left(m_{i}^{2}-\xi^{2}\right)+\left(b_{2} b_{5} s^{2}+b_{3} b_{6} s^{2}\right)} \quad(i=1,2,3) .
\end{gathered}
$$

The displacement components $\widetilde{\bar{u}}_{1}$ and $\widetilde{\bar{u}}_{3}$ are obtained with the aid of (7)-(9) and (12), (13) as

$$
\widetilde{\bar{u}}_{1}=-B_{1} i \xi e^{-m_{1} x_{3}}-B_{2} i \xi e^{-m_{2} x_{3}}-B_{3} i \xi e^{-m_{3} x_{3}}+B_{4} m_{4} e^{-m_{4} x_{3}},
$$




$$
\widetilde{\bar{u}}_{3}=-B_{1} m_{1} e^{-m_{1} x_{3}}-B_{2} m_{2} e^{-m_{2} x_{3}}-B_{3} m_{3} e^{-m_{3} x_{3}}-B_{4} i \xi e^{-m_{4} x_{3}} .
$$

Boundary Conditions and Solution of the Problem. The boundary conditions at $x_{3}=0$ are

$$
\begin{aligned}
\sigma_{33} & =-F_{1} F\left(x_{1}\right) H(t), \quad \sigma_{31}=-F_{2} F\left(x_{1}\right) H(t), \\
p & =F_{3} F\left(x_{1}\right) H(t), \quad \frac{\partial T}{\partial x_{3}}=F_{4} F\left(x_{1}\right) H(t),
\end{aligned}
$$

where $F_{1}$ and $F_{2}$ are the magnitudes of the vertical and horizontal forces, respectively, $F_{3}$ is the constant pressure applied on the boundary, $F_{4}$ is the constant temperature applied on the boundary, $F\left(x_{1}\right)$ is a known function, and $H(t)$ is the Heaviside unit step function.

Applying Laplace and Fourier transforms defined by (8) and (9) on (18) and with the aid of (4), (5), and (10), and

$$
F_{i}^{\prime}=\frac{F_{i}}{\beta T_{0}} \quad(i=1,2,3) \quad \text { and } \quad F_{4}^{\prime}=\frac{F_{4} c_{1}}{\omega^{*} T_{0}},
$$

we obtain

$$
\begin{gathered}
\widetilde{\bar{\sigma}}_{33}=-F_{1} \widetilde{\bar{F}}(\xi) / s, \quad \tilde{\bar{\sigma}}_{31}=-F_{2} \widetilde{\bar{F}}(\xi) / s, \quad \tilde{\bar{p}}=F_{3} \tilde{\bar{F}}(\xi) / s, \\
\frac{\partial T}{\partial x_{3}}=F_{4} \widetilde{\bar{F}}(\xi) / s \quad \text { at } \quad x_{3}=0,
\end{gathered}
$$

where

$$
\begin{gathered}
\widetilde{\bar{\sigma}}_{33}=-R_{1} i \xi \widetilde{\bar{u}}_{1}+R_{2} \frac{d \tilde{\bar{u}}_{3}}{d x_{3}}-\alpha \widetilde{\bar{p}}-\beta \widetilde{\bar{T}}, \\
\widetilde{\bar{\sigma}}_{31}=R_{3}\left[\frac{d \widetilde{\bar{u}}_{1}}{d x_{3}}-i \xi \widetilde{\bar{u}}_{3}\right],
\end{gathered}
$$

and

$$
R_{1}=\frac{\lambda}{\rho c_{1}^{2}}, R_{2}=\frac{\lambda+2 \mu}{\rho c_{1}^{2}}, R_{3}=\frac{\mu}{\rho c_{1}^{2}}
$$

Case 1: For normal force $F_{2}=F_{3}=F_{4}=0$.

Case 2: For tangential force $F_{1}=F_{3}=F_{4}=0$.

Case 3: For pressure source $F_{1}=F_{2}=F_{4}=0$.

Case 4: For thermal source $F_{1}=F_{2}=F_{3}=0$.

Substituting the values of $\widetilde{\bar{u}}_{1}, \widetilde{\bar{u}}_{3}, \widetilde{\bar{p}}$, and $\widetilde{\bar{T}}$ from (16), (17), and (12) in the boundary condition (19) and with help of (20) and (21), after some simplifications, we obtain

$$
\begin{aligned}
& \widetilde{\bar{\sigma}}_{33}=\frac{1}{s \Delta} \widetilde{\bar{F}}(\xi)\left[d_{1} \Delta_{1} e^{-m_{1} x_{3}}+d_{2} \Delta_{2} e^{-m_{2} x_{3}}+d_{3} \Delta_{3} e^{-m_{3} x_{3}}+d_{4} \Delta_{4} e^{-m_{4} x_{3}}\right], \\
& \widetilde{\bar{\sigma}}_{31}=\frac{1}{s \Delta} \widetilde{\bar{F}}(\xi)\left[d_{5} \Delta_{1} e^{-m_{1} x_{3}}+d_{6} \Delta_{2} e^{-m_{2} x_{3}}+d_{7} \Delta_{3} e^{-m_{3} x_{3}}+d_{8} \Delta_{4} e^{-m_{4} x_{3}}\right],
\end{aligned}
$$




$$
\begin{aligned}
& \widetilde{\bar{p}}=\frac{1}{s \Delta} \widetilde{\bar{F}}(\xi)\left[r_{1} \Delta_{1} e^{-m_{1} x_{3}}+r_{2} \Delta_{2} e^{-m_{2} x_{3}}+r_{3} \Delta_{3} e^{-m_{3} x_{3}}\right], \\
& \widetilde{\bar{T}}=\frac{1}{s \Delta} \widetilde{\bar{F}}(\xi)\left[s_{1} \Delta_{1} e^{-m_{1} x_{3}}+s_{2} \Delta_{2} e^{-m_{2} x_{3}}+s_{3} \Delta_{3} e^{-m_{3} x_{3}}\right],
\end{aligned}
$$

where

$$
\begin{gathered}
\Delta=d_{1} d_{8}\left(-m_{3} r_{2} s_{3}+m_{2} r_{3} s_{2}\right)-d_{2} d_{8}\left(-m_{3} r_{1} s_{3}+m_{1} r_{3} s_{1}\right)+d_{3} d_{8}\left(-m_{2} r_{1} s_{2}+m_{1} r_{2} s_{1}\right)- \\
-d_{4} d_{5}\left(-m_{3} r_{2} s_{3}+m_{2} r_{3} s_{2}\right)-d_{4} d_{6}\left(-m_{1} r_{3} s_{1}+m_{3} r_{1} s_{3}\right)-d_{4} d_{7}\left(-m_{2} r_{1} s_{2}+m_{1} r_{2} s_{1}\right), \\
d_{i}=-R_{1} \xi^{2}+R_{2} m_{i}^{2}-\alpha r_{i}-s_{i} \quad(i=1,2,3), \quad d_{4}=i \xi m_{4}\left(-R_{1}+R_{2}\right), \\
d_{j}=2 i \xi m_{j} R_{3} \quad(j=5,6,7), \quad d_{8}=-R_{3}\left(m_{4}^{2}+\xi^{2}\right),
\end{gathered}
$$

and $\Delta_{1}, \Delta_{2}, \Delta_{3}$, and $\Delta_{4}$ are obtained by replacing $\left[-F_{1},-F_{2}, F_{3}, F_{4}\right]^{T}$ in $\Delta$.

\section{Applications.}

Case 1. Concentrated Source. The values for displacement, stress, pore pressure and temperature change presented in Eqs. (22)-(25) will used to yield the response over the half space subjected to a concentrated source as

$$
F\left(x_{1}\right)=\delta\left(x_{1}\right),
$$

where $\delta(\cdot)$ is the Dirac delta function.

Applying the Laplace and Fourier transforms defined by (8) and (9) on (26), yield

$$
\widetilde{\bar{F}}(\xi)=1 \text {. }
$$

The component of displacement, stress, pore pressure, and temperature change are obtained by using (27) in (22)-(25).

Case 2. Influence Function. Here

$$
F\left(x_{1}\right)=\Psi_{1}\left(x_{1}\right),
$$

where $\Psi_{1}\left(x_{1}\right)$ is a known function, which can have two types of values:

1. Uniformly distributed source,

$$
\Psi_{1}\left(x_{1}\right)=\left\{\begin{array}{ll}
1, & \left|x_{1}\right| \leq a \\
0, & \left|x_{1}\right|>a
\end{array}\right\},
$$

where $2 a$ is non-dimensional width of the strip.

Applying the Laplace and Fourier transforms defined by (8) and (9) on (28) and (29), we obtain

$$
\widetilde{\bar{F}}(\xi)=(2 \sin \xi a) / \xi
$$

2. Linearly distributed source,

$$
\Psi_{1}\left(x_{1}\right)=\left\{\begin{array}{cc}
1-\left|x_{1}\right| / a, & \left|x_{1}\right| \leq a \\
0, & \left|x_{1}\right|>a
\end{array}\right\} .
$$


Applying the Laplace and Fourier transforms defined by (8) and (9) on (28) and (31), we obtain

$$
\widetilde{F}(\xi, s)=\frac{2[1-\cos (\xi a)]}{\xi^{2} a} .
$$

The expression for stresses, pore pressure and temperature field are obtained for concentrated, uniformly and linearly distributed sources by replacing $\widetilde{\bar{F}}(\xi)$ from (27), (30), and (32) in (22)-(25).

Special Case. In the absence of porosity effect, the boundary conditions reduce to

$$
\tilde{\bar{\sigma}}_{33}=-F_{1} \tilde{\bar{F}}(\xi) / s, \quad \widetilde{\bar{\sigma}}_{31}=-F_{2} \tilde{\bar{F}}(\xi) / s, \quad \frac{\partial T}{\partial x_{3}}=F_{4} \tilde{\bar{F}}(\xi) / s
$$

and we obtain the corresponding expressions for stress components and temperature field in thermoelastic half space are obtained as

$$
\begin{gathered}
\widetilde{\bar{\sigma}}_{z z}=\frac{1}{s \Delta_{10}} \tilde{\bar{F}}(\xi)\left[d_{9} \Delta_{5} e^{-m_{5} x_{3}}+d_{10} \Delta_{6} e^{-m_{6} x_{3}}+d_{4} \Delta_{7} e^{-m_{4} x_{3}}\right], \\
\widetilde{\bar{\sigma}}_{z r}=\frac{1}{s \Delta_{10}} \widetilde{\bar{F}}(\xi)\left[d_{11} \Delta_{5} e^{-m_{5} x_{3}}+d_{12} \Delta_{6} e^{-m_{6} x_{3}}+d_{8} \Delta_{7} e^{-m_{4} x_{3}}\right], \\
\widetilde{\bar{T}}=\frac{1}{s \Delta_{10}} \tilde{\bar{F}}(\xi)\left[r_{5} \Delta_{5} e^{-m_{5} x_{3}}+r_{6} \Delta_{6} e^{-m_{6} x_{3}}\right],
\end{gathered}
$$

where

$$
\begin{aligned}
& d_{9}=-R_{1} \xi^{2}+R_{2} m_{5}^{2}-r_{5}, \quad d_{10}=-R_{1} \xi^{2}+R_{2} m_{6}^{2}-r_{6}, \quad d_{11}=2 i \xi m_{5} R_{3}, \\
& d_{12}=2 i \xi m_{6} R_{3}, \quad \Delta_{10}=d_{9} d_{8} m_{6} r_{6}-d_{10} d_{8} m_{5} r_{5}-d_{4} d_{11} m_{6} r_{6}+d_{4} d_{12} m_{5} r_{5},
\end{aligned}
$$

and $\Delta_{5}, \Delta_{6}$, and $\Delta_{7}$ are obtained by replacing $\left[-F_{1},-F_{2}, F_{4}\right]^{T}$ in $\Delta_{10}$.

Taking $F_{2}=F_{4}=0, F_{1}=F_{4}=0$, and $F_{1}=F_{2}=0$ in Eqs. (34), (35), and (36), respectively, we obtain the stress components and temperature change for normal force, tangential forces, and thermal source, respectively.

Inversion of the Transform. The transformed displacements, stresses, pore pressure and temperature field are functions of the parameters of the Laplace and Fourier transforms $s$ and $\xi$, respectively, and hence are of the form $\widetilde{\widetilde{f}}\left(\xi, x_{3}, s\right)$. To obtain the solution of the problem in the physical domain, we invert the Laplace and Fourier transforms by using the method described by Kumar and Rani [31].

Numerical Results and Discussion. Following Jabbari and Dehbani [30], for numerical computation, we take the value of the various physical parameters as $E=6 \cdot 10^{5} \mathrm{MPa}$, $v=0.3, T_{0}=293 \mathrm{~K}, K_{s}=2 \cdot 10^{10}, K_{w}=5 \cdot 10^{9}, K=0.5, \alpha_{s}=1.5 \cdot 10^{-5}, \alpha_{W}=2 \cdot 10^{-4}$, $c_{s}=0.8, c_{w}=4.2, \rho_{s}=2.6 \cdot 10^{6}, \rho_{w}=1 \cdot 10^{6}, \alpha=1, F_{1}=F_{2}=F_{3}=F_{4}=1$.

The values of normal stress $\sigma_{33}$, tangential stress $\sigma_{31}$, pore pressure $p$, and temperature change $T$ for incompressible fluid saturated thermoporoelastic medium (FSPM) and empty porous thermoelastic medium (EPM) are shown due to concentrated source (CS), uniformly distributed source (UDS), and linearly distributed source (LDS). The 
computation are carried out for two values of dimensionless time $t=0.01$ and 0.05 at $x_{3}=1$ for the range $0 \leq x_{1} \leq 10$, and $a=1$.

The solid lines either with or with central symbols represents the variations for $t=0.01$, whereas the dashed lines with or without central symbols represents the variations for $t=0.05$. Curves without central symbols correspond to the case of FSPM whereas those with central symbols corresponds to the case of EPM.

Figure 2a shows the variation of normal stress component $\sigma_{33}$ w.r.t distance $x_{1}$ for both FSPM and EPM due to concentrated normal force. The value of $\sigma_{33}$ start with initial decrease and remain close to the boundary surface for FSPM as $x_{1}$ increases and in case of EPM, its value decrease in the range $0 \leq x \leq 3$ and then oscillates as $x_{1}$ increases for both values of time.

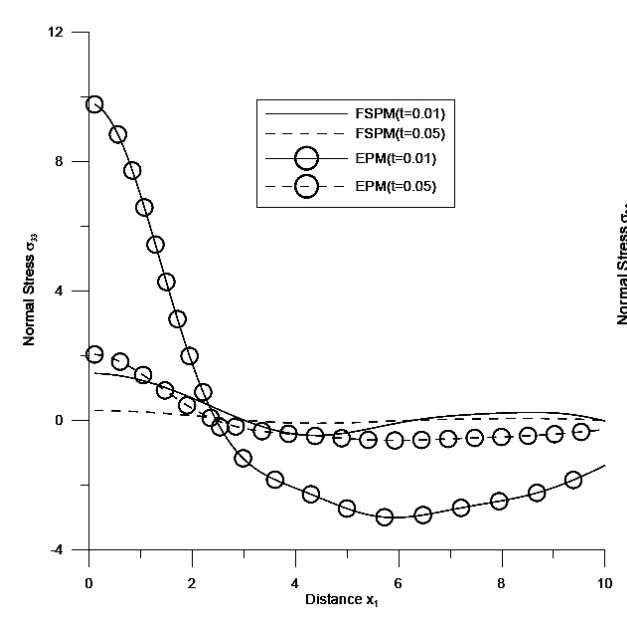

a

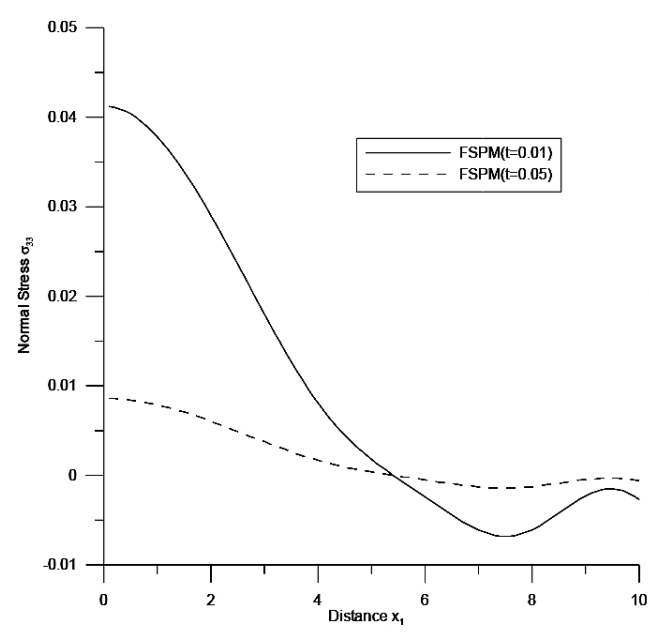

$\mathrm{c}$

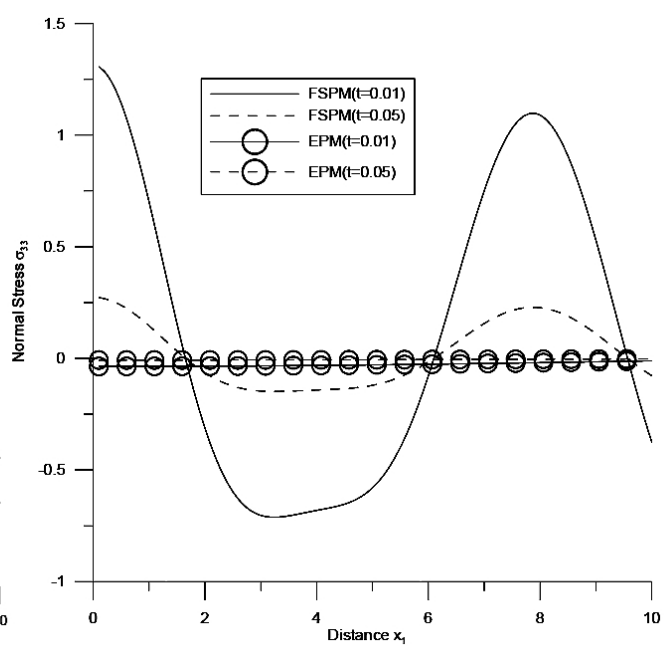

b

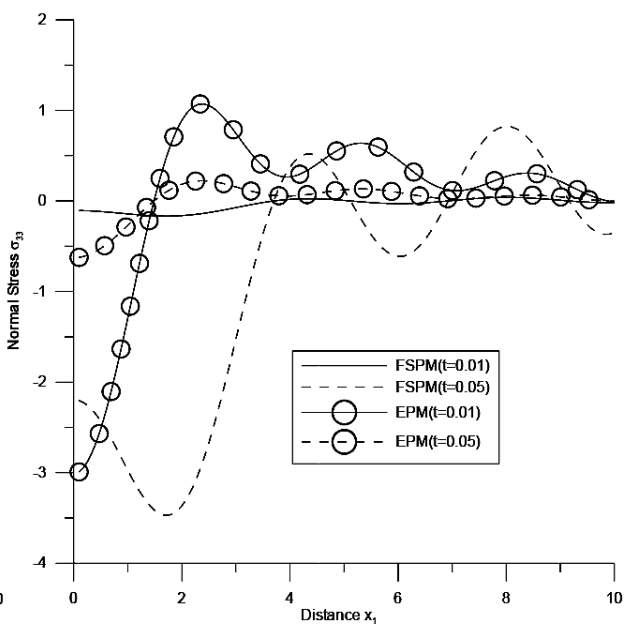

d

Fig. 2. Variation of normal stress $\sigma_{33}$ with distance $x_{1}$ due to concentrated normal force (a), concentrated tangential force (b), concentrated pressure source (c), and thermal source (d).

Figure $2 \mathrm{~b}$ shows the variation of normal stress component $\sigma_{33}$ w.r.t distance $x_{1}$ for both FSPM and EPM due to concentrated tangential force. The value of $\sigma_{33}$ first decrease 
in the range $0 \leq x_{1} \leq 4$ and then starts oscillating for FSPM as $x_{1}$ increases for both values of time whereas in case of EPM, the value of $\sigma_{33}$ remain close to the boundary surface for different values of time as $x_{1}$ increases.

Figure 2c shows the variation of normal stress component $\sigma_{33}$ w.r.t distance $x_{1}$ for FSPM due to concentrated pressure source. The value of $\sigma_{33}$ decrease in the range $0 \leq x_{1} \leq 7.5$ and then starts increasing for different values of time.

Figure $2 \mathrm{~d}$ shows the variation of normal stress component $\sigma_{33}$ w.r.t distance $x_{1}$ for FSPM and EPM due to thermal source. The value of $\sigma_{33}$ oscillates for different value of time for FSPM as $x_{1}$ increases whereas in case of EPM, the value of $\sigma_{33}$ first increases in the range $0 \leq x_{1} \leq 2.2$ and then starts oscillating for both values of time.

Figure 3 a shows the variation of pore pressure $p$ w.r.t distance $x_{1}$ for FSPM due to concentrated normal force. Near the application of the source the value of $p$ is more for the time $t=0.05$ as compared to time $t=0.01$ and away from the source reverse behavior is noticed.

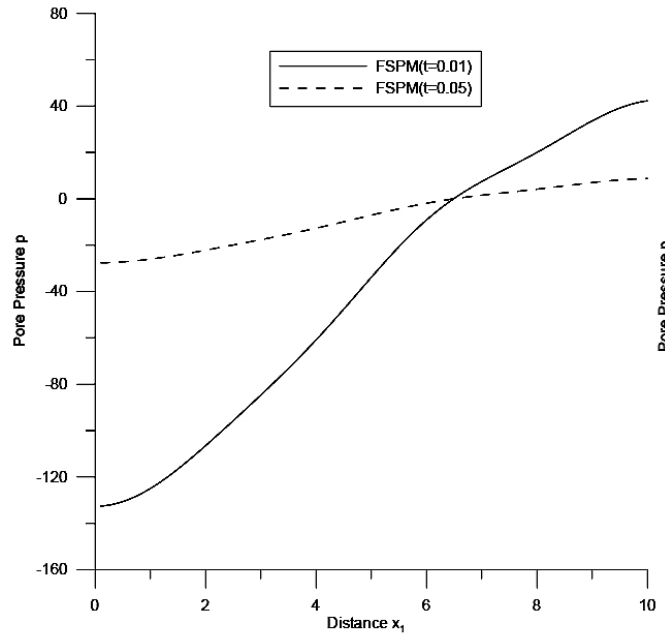

a

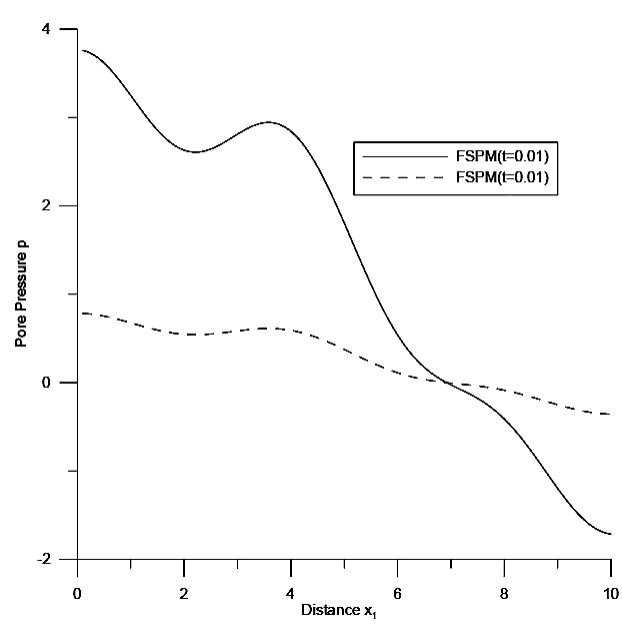

c

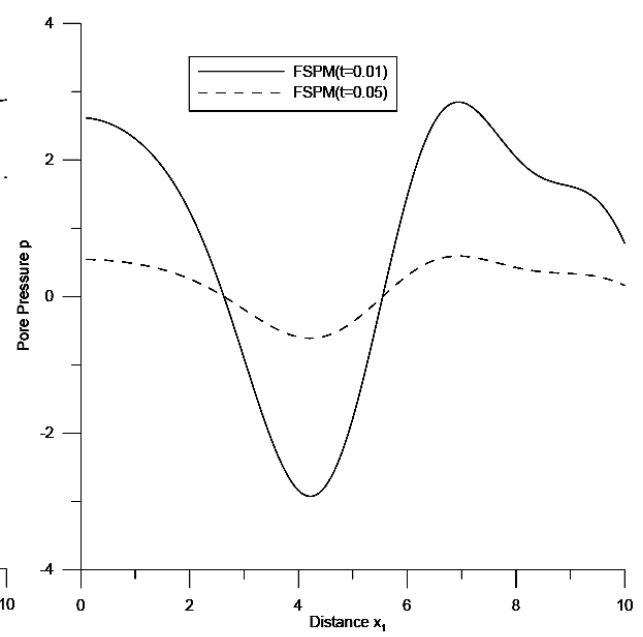

b

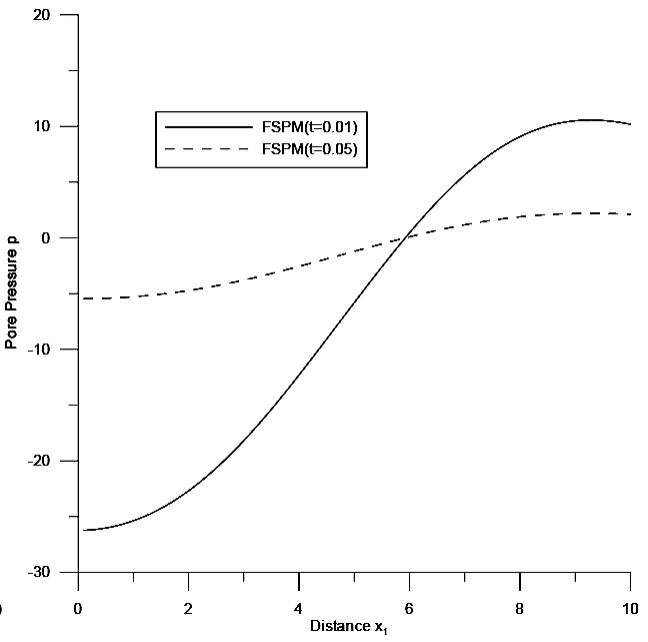

d

Fig. 3. Variation of pore pressure $p$ with distance $x_{1}$ due to concentrated normal force (a), concentrated tangential force (b), concentrated pressure source (c), and thermal source (d). 
Figure $3 \mathrm{~b}$ shows the variation of pore pressure $p$ w.r.t distance $x_{1}$ for FSPM due to concentrated tangential force. The value of $p$ first decreases in the range $0 \leq x_{1} \leq 4.2$ and then starts increasing for different values of time.

Figure $3 \mathrm{c}$ shows the variation of pore pressure $p$ w.r.t distance $x_{1}$ for FSPM due to concentrated pressure source. The value of $p$ starts decreasing as $x_{1}$ increases for different values of time.

Figure $3 \mathrm{~d}$ shows the variation of pore pressure $p$ w.r.t distance $x_{1}$ for FSPM due to thermal source. For the time $t=0.05$ the value of $p$ is higher in the range $0 \leq x_{1} \leq 6$ whereas for the time $t=0.01$ the value of $p$ is higher in the range $6 \leq x_{1} \leq 10$.

Figure 4a shows the variation of temperature $T$ w.r.t distance $x_{1}$ for FSPM and EPM due to concentrated normal force. The value of $T$ first decreases and then oscillates for FSPM as $x_{1}$ increases for both value of time whereas for EPM it first increases in the range $0 \leq x_{1} \leq 3.5$ and then oscillates for both values of time.

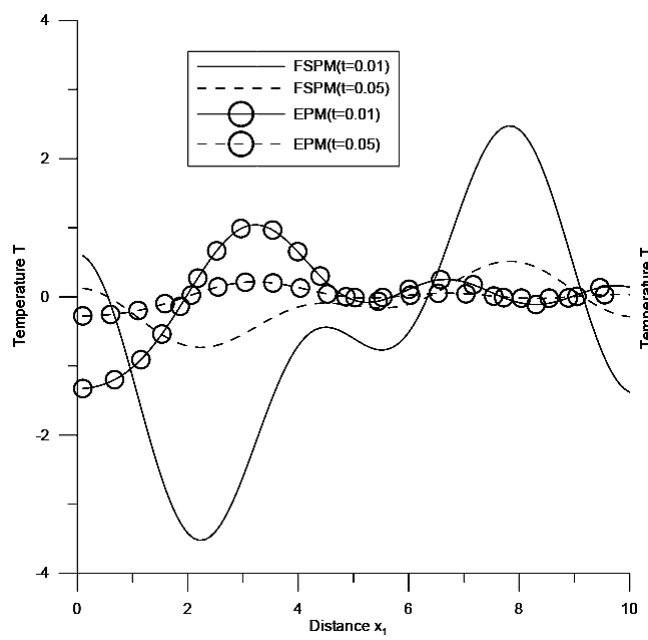

a

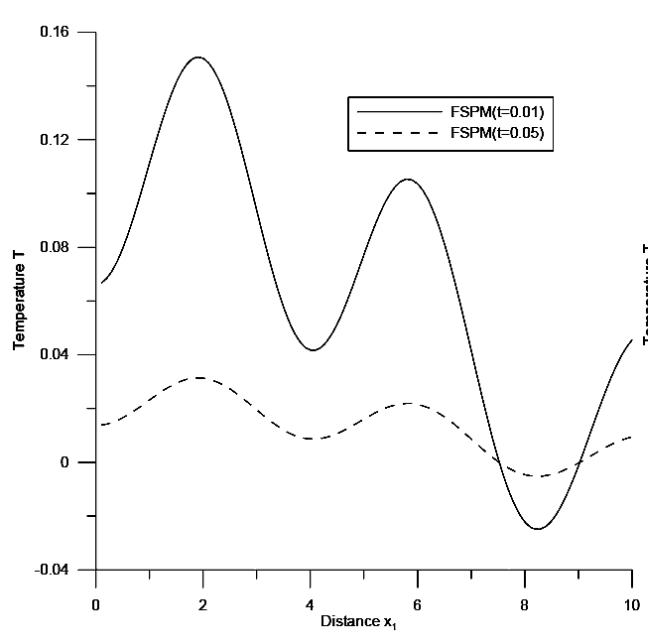

$\mathrm{c}$

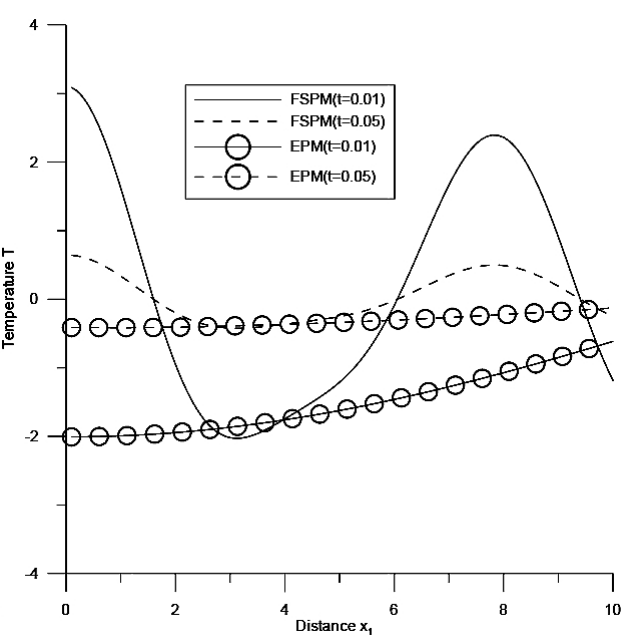

b

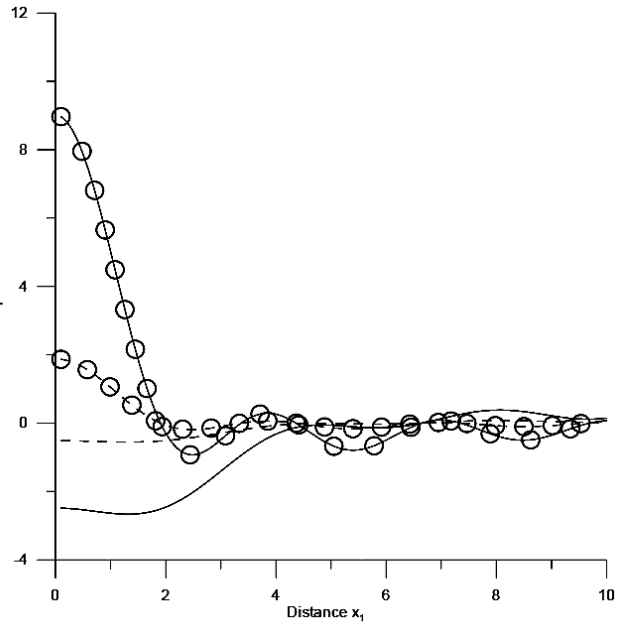

d

Fig. 4. Variation of temperature $T$ with distance $x_{1}$ due to concentrated normal force (a), concentrated tangential force (b), concentrated pressure source (c), and thermal source (d). 
Figure $4 \mathrm{~b}$ shows the variation of temperature $T$ w.r.t distance $x_{1}$ for FSPM and EPM due to tangential force. The value of $T$ first decreases and then oscillates for FSPM and for EPM shows a small increase as $x_{1}$ increases for different values of time.

Figure $4 \mathrm{c}$ shows the variation of temperature $T$ w.r.t distance $x_{1}$ for FSPM due to pressure source. The value of $T$ starts oscillates for the time $t=0.01$ and 0.05 .

Figure $4 \mathrm{~d}$ shows the variation of temperature $T$ w.r.t distance $x_{1}$ for FSPM and EPM due to thermal source. The value of $T$ first increases and remains close to zero for FSPM as $x_{1}$ increases for both value time whereas for EPM the value of $T$ decreases in the range $0 \leq x_{1} \leq 2.5$ and then starts oscillates for both value of time as $x_{1}$ increases.

Figure 5a shows the variation of normal stress component $\sigma_{33}$ w.r.t distance $x_{1}$ for FSPM and EPM due to normal force over uniformly distributed source. The value of $\sigma_{33}$ for FSPM first decreases in the range $0 \leq x_{1} \leq 4.5$ and then oscillates for both value of time whereas for EPM its value shows a small decrease for both values of time as $x_{1}$ increases.

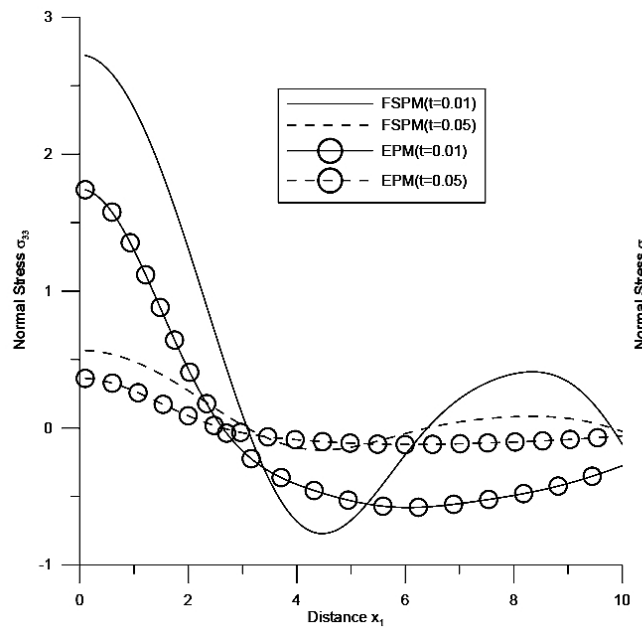

$\mathrm{a}$

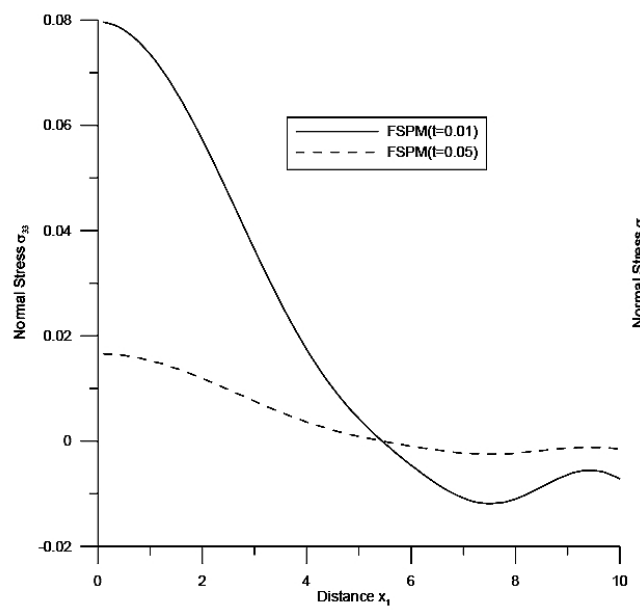

$\mathrm{c}$

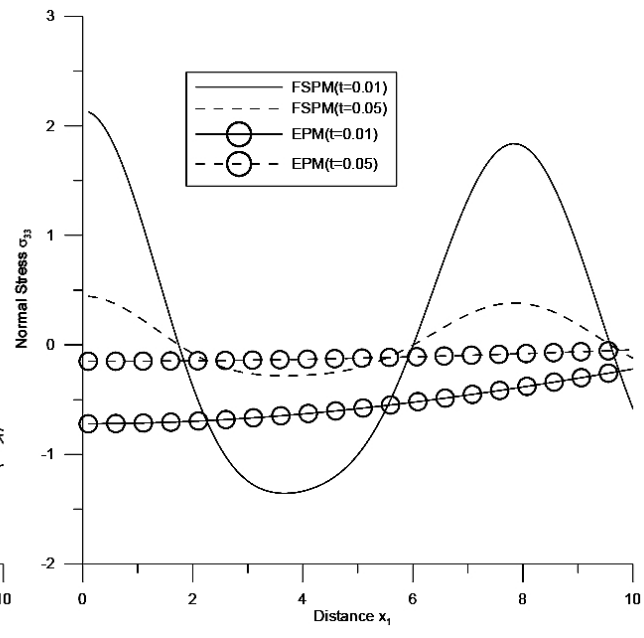

b

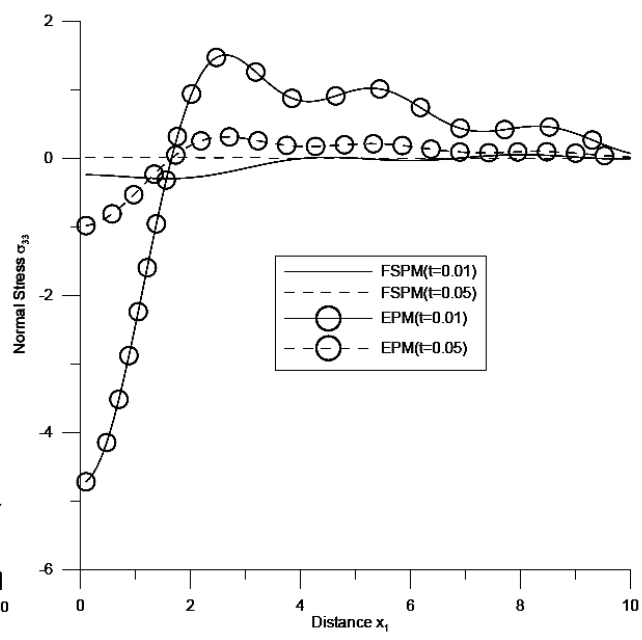

d

Fig. 5. Variation of normal stress $\sigma_{33}$ with distance $x_{1}$ due to concentrated normal force (a), concentrated tangential force (b), concentrated pressure source (c), and thermal source (d). 
Figure $5 \mathrm{~b}$ shows the variation of normal stress component $\sigma_{33}$ w.r.t distance $x_{1}$ for FSPM and EPM due to tangential force over uniformly distributed source. The value of $\sigma_{33}$ first decreases in the range $0 \leq x_{1} \leq 4$ and then oscillates for FSPM as $x_{1}$ increases for both value of time where as for EPM its value shows a small increase as $x_{1}$ increases for different values of time.

Figure 5c shows the variation of normal stress component $\sigma_{33}$ w.r.t distance $x_{1}$ for FSPM due to pressure source over uniformly distributed source. The value of $\sigma_{33}$ shows a sharp decrease in the range $0 \leq x_{1} \leq 7.6$ for FSPM as $x_{1}$ increases for both values of time.

Figure $5 \mathrm{~d}$ shows the variation of normal stress component $\sigma_{33}$ w.r.t distance $x_{1}$ for FSPM and EPM due to thermal source over uniformly distributed source. The value of $\sigma_{33}$ remain close to zero for FSPM as $x_{1}$ increases for both value of time whereas the value of $\sigma_{33}$ first increases in the range $0 \leq x_{1} \leq 2.2$ and then oscillates for EPM as $x_{1}$ increases for the time $t=0.01$ and 0.05 .

Figure 6a shows the variation of pore pressure $p$ w.r.t distance $x_{1}$ for FSPM due to normal force over uniformly distributed source. The value of $p$ shows a sharp increase for FSPM for different values of time as $x_{1}$ increases.

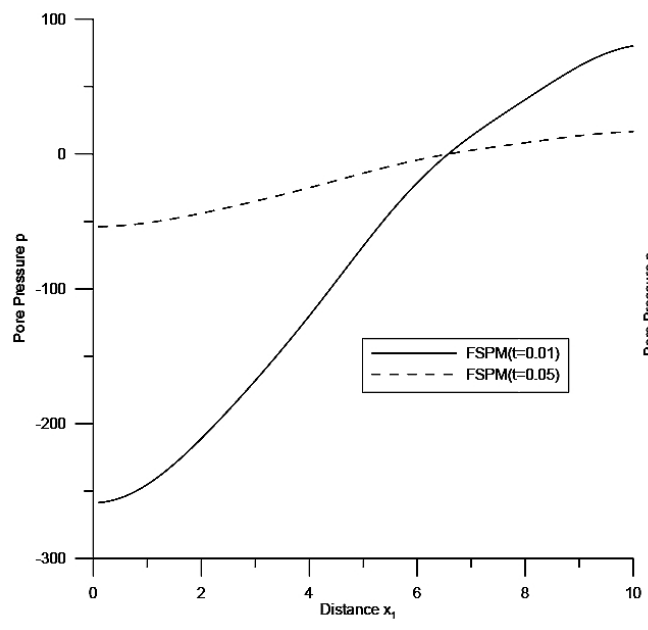

a

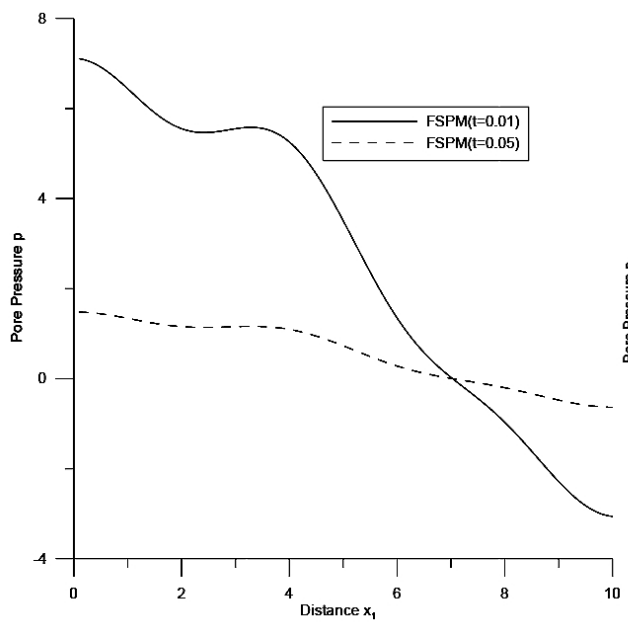

c

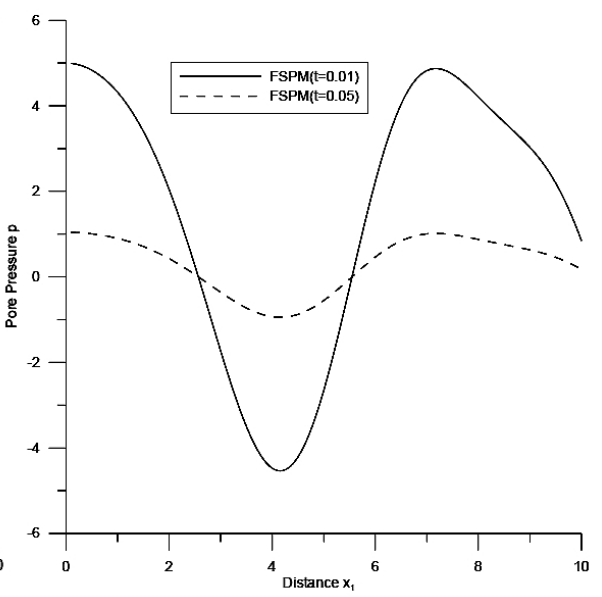

b

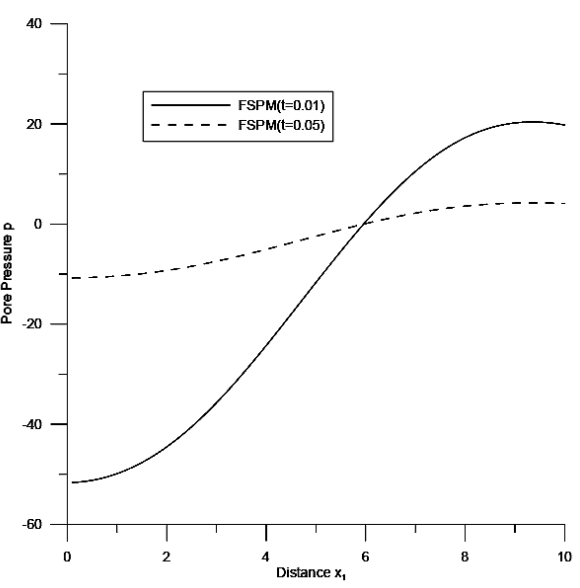

d

Fig. 6. Variation of pore pressure $p$ with distance $x_{1}$ due to concentrated normal force (a), concentrated tangential force (b), concentrated pressure source (c), and thermal source (d). 
Figure $6 \mathrm{~b}$ shows the variation of pore pressure $p$ w.r.t distance $x_{1}$ for FSPM due to tangential force over uniformly distributed source. The value of $p$ first decreases in the range $0 \leq x_{1} \leq 4.2$ and then increases as $x_{1}$ increases for FSPM for the time $t=0.01$ and 0.05 .

Figure $6 \mathrm{c}$ shows the variation of pore pressure $p$ w.r.t distance $x_{1}$ for FSPM due to pressure source over uniformly distributed source. The value of $p$ shows a sharp decrease for FSPM as $x_{1}$ increases for different values of time.

Figure $6 \mathrm{~d}$ shows the variation of pore pressure $p$ w.r.t distance $x_{1}$ for FSPM due to thermal source over uniformly distributed source. For the time $t=0.05$ the value of $p$ is more in the range $0 \leq x_{1} \leq 6$ whereas for the time $t=0.01$ the value of $p$ is more in the range $6 \leq x_{1} \leq 10$.

Figure 7a shows the variation of temperature $T$ w.r.t distance $x_{1}$ for FSPM and EPM due to normal force over uniformly distributed source. The value of $T$ first decreases and then oscillates for FSPM whereas for EPM the value of $T$ first increases and then oscillates as $x_{1}$ increases for different values of time.

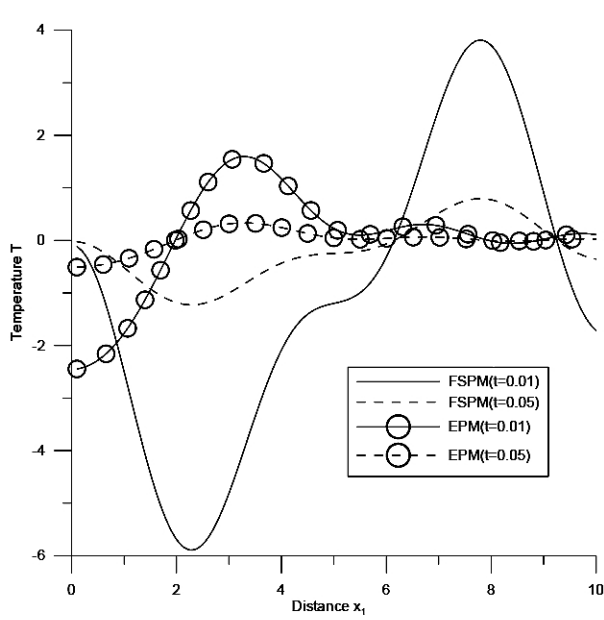

a

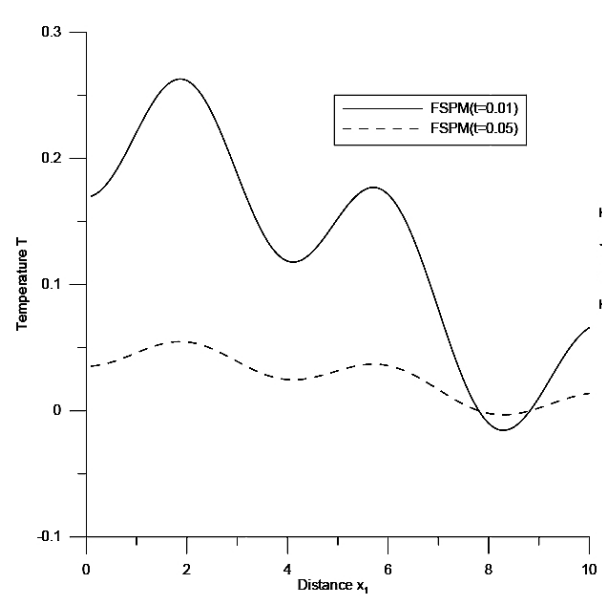

$\mathrm{c}$

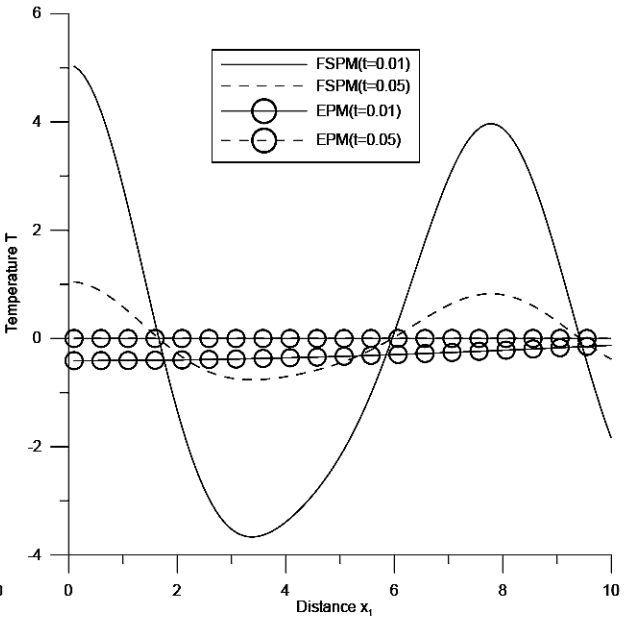

b

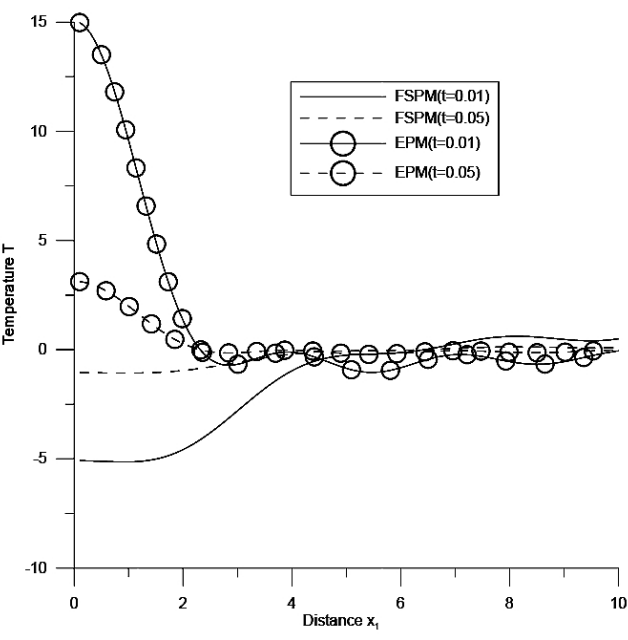

d

Fig. 7. Variation of temperature $T$ with distance $x_{1}$ due to concentrated normal force (a), concentrated tangential force (b), concentrated pressure source (c), and thermal source (d). 
Figure $7 \mathrm{~b}$ shows the variation of temperature $T$ w.r.t distance $x_{1}$ for FSPM and EPM due to tangential force over uniformly distributed source. The value of $T$ first decreases and then start oscillates for FSPM and remain close to zero for EPM as $x_{1}$ increases for both values time.

Figure 7c shows the variation of temperature $T$ w.r.t distance $x_{1}$ for FSPM due to pressure source over uniformly distributed source. The value of $T$ first increases and then starts decreasing in oscillatory manner for FSPM as $x_{1}$ increases for the time $t=0.01$ and 0.05 .

Figure $7 \mathrm{~d}$ shows the variation of temperature $T$ w.r.t distance $x_{1}$ for FSPM and EPM due to thermal source over uniformly distributed source. The value of $T$ first increases and remains close to zero for FSPM as $x_{1}$ increases for both value time whereas for EPM the value of $T$ decreases in the range $0 \leq x_{1} \leq 2.5$ and then oscillates for both value of time as $x_{1}$ increases.

Figure 8a shows the variation of normal stress component $\sigma_{33}$ w.r.t distance $x_{1}$ for FSPM and EPM due to normal force over linearly distributed source. The value of $\sigma_{33}$ remain close to zero for FSPM whereas for EPM it decreases in the range $0 \leq x_{1} \leq 4$ and then becomes linear for both values of time as $x_{1}$ increases.

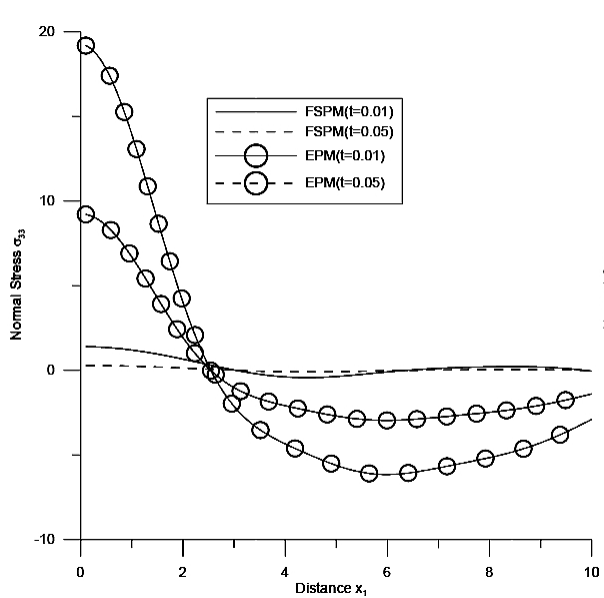

a

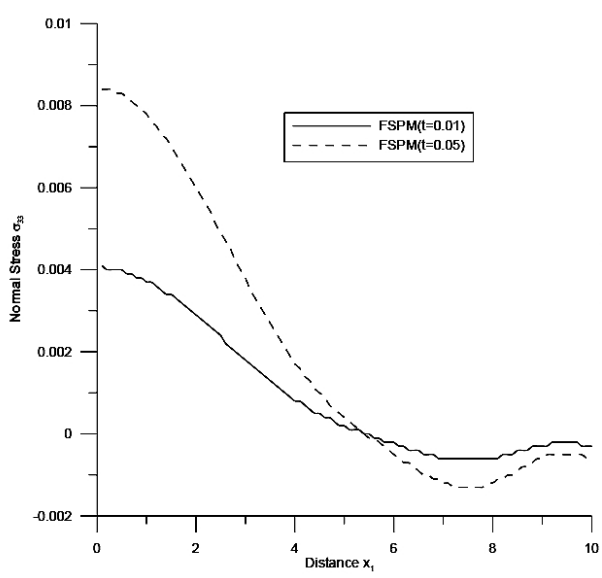

c

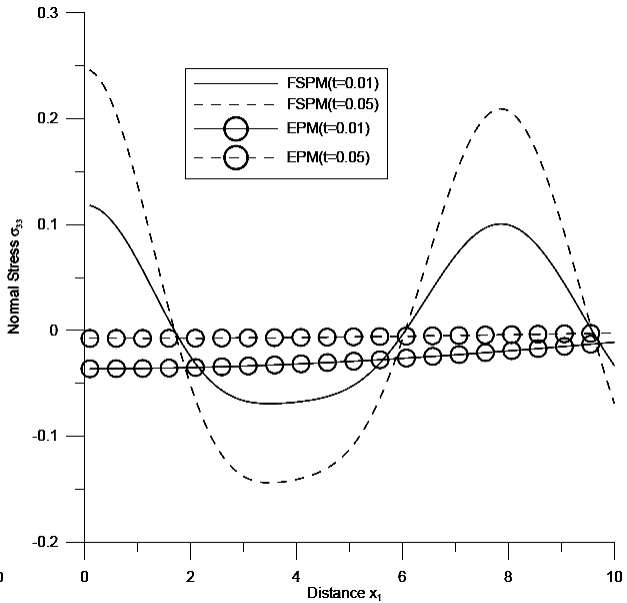

$\mathrm{b}$

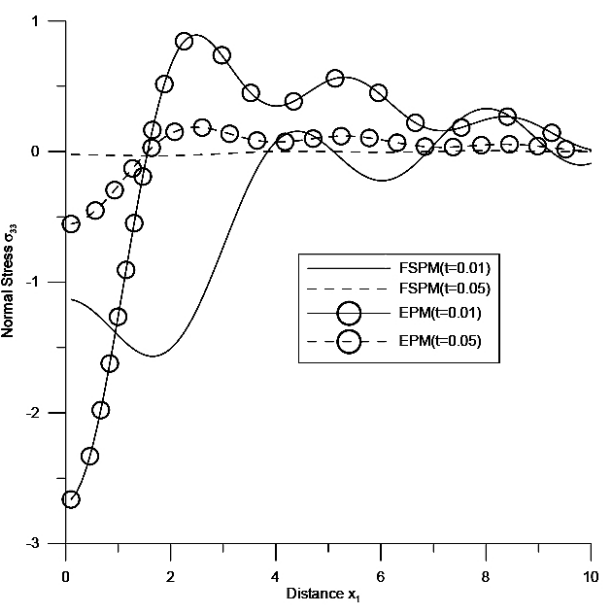

d

Fig. 8. Variation of normal stress $\sigma_{33}$ with distance $x_{1}$ due to concentrated normal force (a), concentrated tangential force (b), concentrated pressure source (c), and thermal source (d). 
Figure $8 \mathrm{~b}$ shows the variation of normal stress component $\sigma_{33}$ w.r.t distance $x_{1}$ for FSPM and EPM due to tangential force over linearly distributed source. The value of $\sigma_{33}$ for FSPM first decreases in the range $0 \leq x_{1} \leq 4$, increases in the range $4 \leq x_{1} \leq 8$ whereas for EPM it remains close to zero for different values of time as $x_{1}$ increases.

Figure 8c shows the variation of normal stress component $\sigma_{33}$ w.r.t distance $x_{1}$ for FSPM due to pressure source over linearly distributed source. The value of $\sigma_{33}$ shows a sharp decrease in the range $0 \leq x_{1} \leq 7.8$ and then increases for FSPM as $x_{1}$ increases for different values of time.

Figure $8 \mathrm{~d}$ shows the variation of normal stress component $\sigma_{33}$ w.r.t distance $x_{1}$ for FSPM and EPM due to thermal source over linearly distributed source. The value of $\sigma_{33}$ oscillates for FSPM whereas the value of $\sigma_{33}$ first increases in the range $0 \leq x_{1} \leq 2$ and then oscillates for EPM as $x_{1}$ increases for both values of time.

Figure 9a shows the variation of pore pressure $p$ w.r.t distance $x_{1}$ for FSPM due to normal force over linearly distributed source. The value of $p$ shows a sharp increase for FSPM as $x_{1}$ increases for time $t=0.01$ and 0.05 .

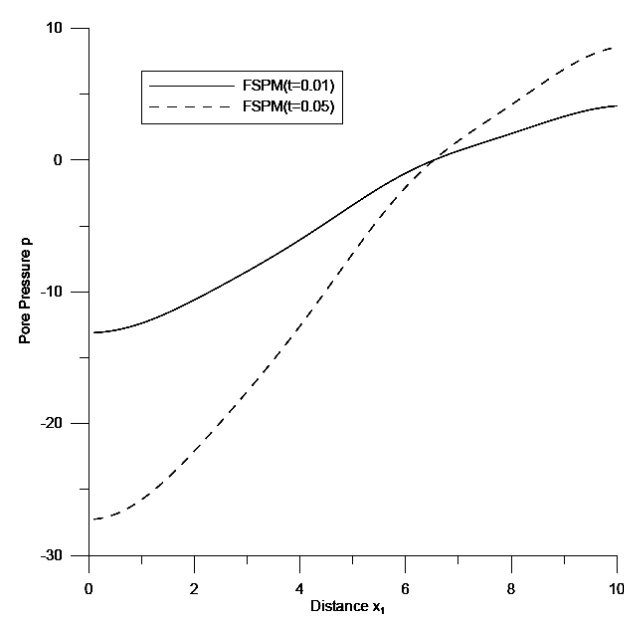

a

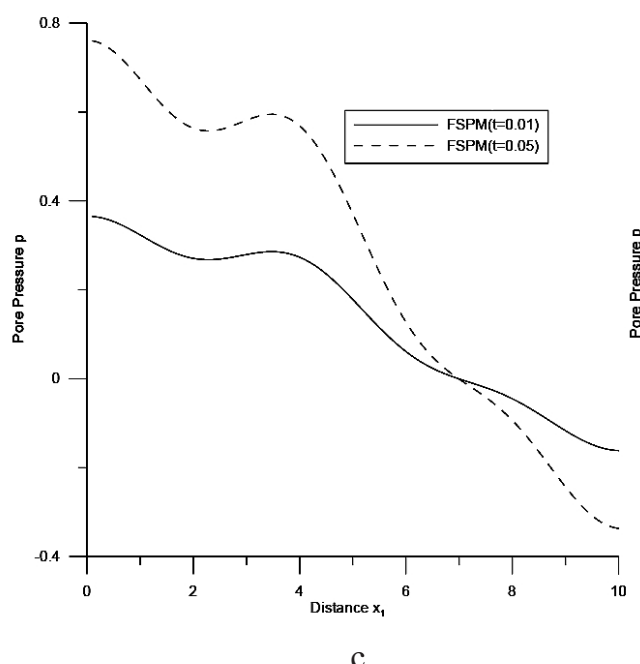

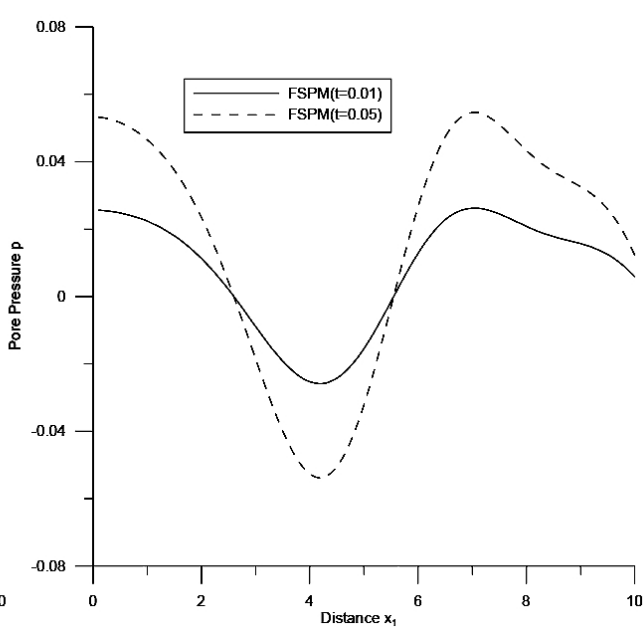

b

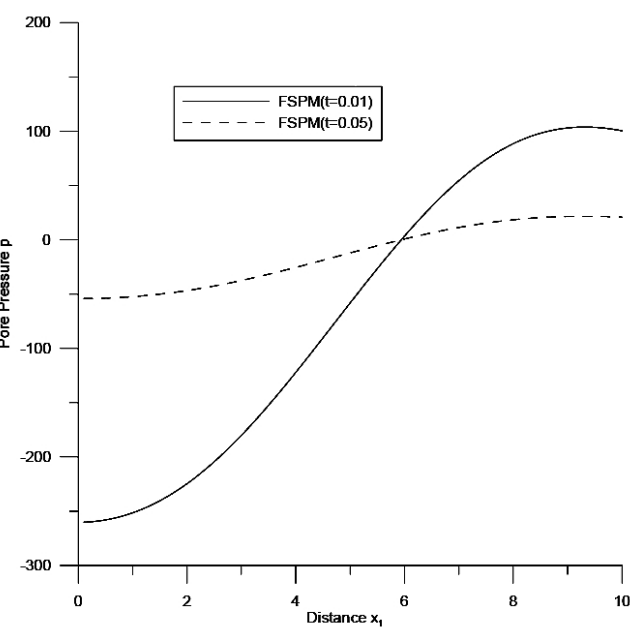

d

Fig. 9. Variation of pore pressure $p$ with distance $x_{1}$ due to concentrated normal force (a), concentrated tangential force (b), concentrated pressure source (c), and thermal source (d). 
Figure $9 \mathrm{~b}$ shows the variation of pore pressure $p$ w.r.t distance $x_{1}$ for FSPM due to tangential force over linearly distributed source. The value of $p$ first decreases in the range $0 \leq x_{1} \leq 4.2$ and then starts increasing for different values of time.

Figure 9c shows the variation of pore pressure $p$ w.r.t distance $x_{1}$ for FSPM due to pressure source over linearly distributed source. The value of $p$ starts decreasing as $x_{1}$ increases for different values of time.

Figure $9 \mathrm{~d}$ shows the variation of pore pressure $p$ w.r.t distance $x_{1}$ for FSPM due to thermal source over linearly distributed source. The value of $p$ starts increasing as $x_{1}$ increases for both values of time.

Figure 10a shows the variation of temperature $T$ w.r.t distance $x_{1}$ for FSPM and EPM due to normal force over linearly distributed source. The value of $T$ first decreases and then oscillates for FSPM as $x_{1}$ increases for both value of time whereas for EPM it remain close to zero for both values of time.

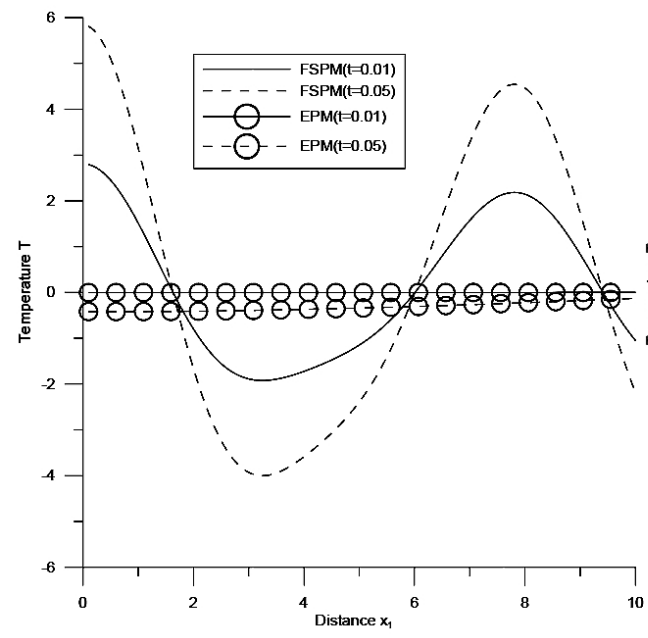

$\mathrm{a}$

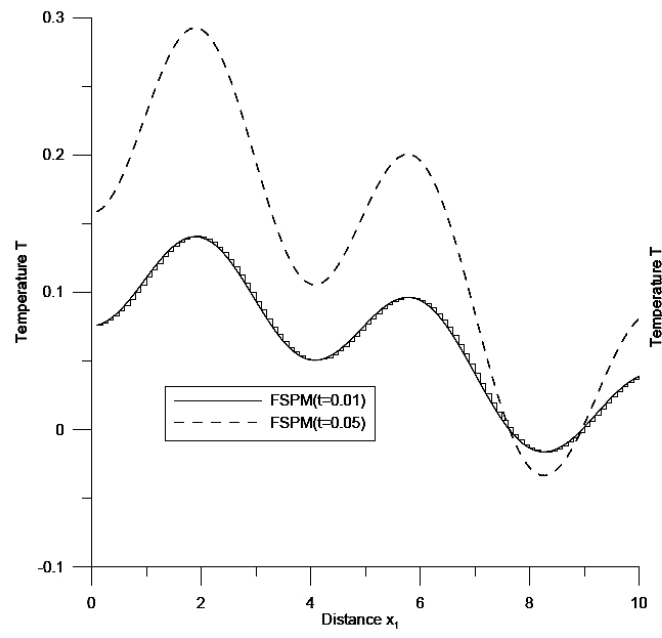

$\mathrm{c}$

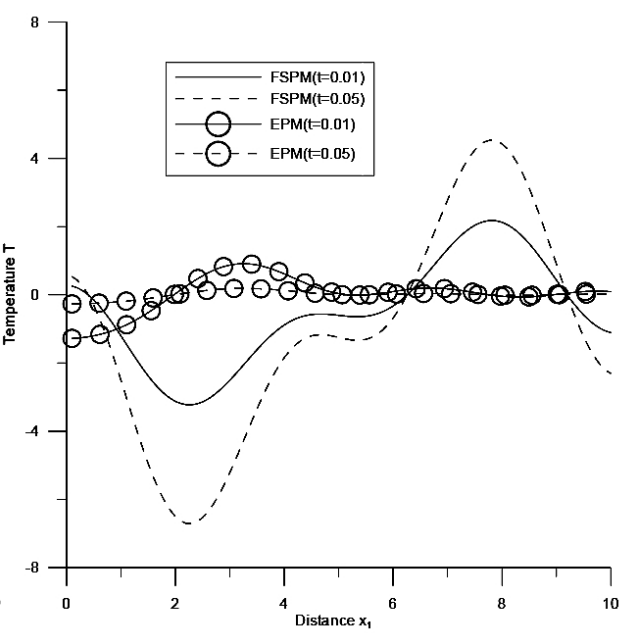

b

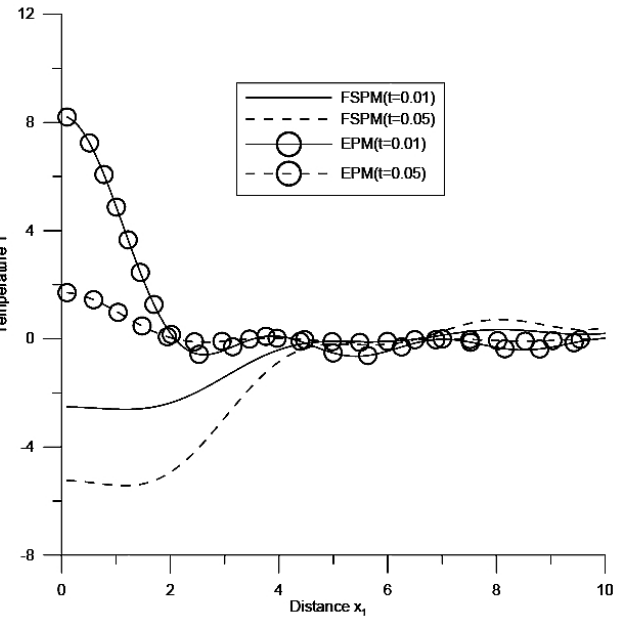

d

Fig. 10. Variation of temperature $T$ with distance $x_{1}$ due to concentrated normal force (a), concentrated tangential force (b), concentrated pressure source (c), and thermal source (d). 
Figure 10b shows the variation of temperature $T$ w.r.t distance $x_{1}$ for FSPM and EPM due to tangential force over linearly distributed source. The value of $T$ oscillates for FSPM and for EPM it remains close to zero in oscillatory manner as $x_{1}$ increases for different values of time.

Figure 10c shows the variation of temperature $T$ w.r.t distance $x_{1}$ for FSPM due to pressure source over linearly distributed source. The value of $T$ first increases and then starts decreasing for FSPM as $x_{1}$ increases for the time $t=0.01$ and 0.05 .

Figure $10 \mathrm{~d}$ shows the variation of temperature $T$ w.r.t distance $x_{1}$ for FSPM and EPM due to thermal source over linearly distributed source. The value of $T$ first shows a small increase and then oscillates for FSPM whereas for EPM value of $T$ first decreases in the range $0 \leq x_{1} \leq 2.8$ and then becomes linear as $x_{1}$ increases for different values of time.

Conclusions. The components of displacement, stress, pore pressure and temperature change are obtained due to the various sources by using the Laplace and Fourier techniques. The values of the components of displacement, stress, pore pressure and temperature are close to each other due to CS, UDS and LDS. Near the application of the source, the porosity effect decreases the values of $\sigma_{33}$ for normal force, pressure source and thermal source where as it decreases the values of $p$ for normal force and tangential force but increases the values for thermal source.

\section{Резюме}

Досліджено двовимірну задачу щодо виникнення збурень від механічних і термічних джерел в однорідному теплопровідному пористо-пружному середовищі. При розв'язанні цієї задачі використовували перетворення Лапласа і Фур'є. Для ілюстрації перспективності даного підходу розглянуто різні випадки з точковими і розподіленими джерелами. За допомогою методики числової інверсії виконано перетворення компонент переміщень, напружень, тиску в порах і температурних флуктуацій. Вплив пористості на вказані параметри представлено у вигляді графіків. Розглянуто деякі спеціальні випадки, що мають практичний інтерес.

1. K. von Terzaghi, "Die Berechnug der Durchlässigkeit des Tones aus dem Verlauf der hydromechanischen Spannungserscheinungen," Sitzungsbericht der Akademie der Wissenschaften (Wien): Mathematisch-Naturwissenschaftlichen Klasse, 132, 125-138 (1923).

2. K. von Terzaghi, Erdbaumechanik auf bodenphysikalischer Grundlage, Franz Deuticke, Leipzig-Wien (1925), p. 399.

3. M. A. Biot, "General theory of three dimensional consolidation," J. Appl. Phys., 12, No. 2, 155-161 (1941).

4. M. A. Biot, "Theory of propagation of elastic waves in fluid saturated porous solid. I. Low frequency range," J. Acoust. Soc. Am., 28, 168-178 (1956).

5. M. A. Biot, "Theory of propagation of elastic waves in fluid saturated porous solid. II. Higher frequency range," J. Acousti. Soc. Am., 28, 179-191 (1956).

6. M. A. Biot, "Mechanics of deformation and acoustic propagation in porous media," $J$. Appl. Phys., 33, No. 4, 1482-1498 (1962).

7. R. Kumar and B. S. Hundal, "The wave propagation in a fluid saturated incompressible porous medium," Indian J. Pure Appl. Math., 34, No. 4, 651-665 (2003).

8. B. Bai and T. Li, "Solution for cylindrical cavety in saturated thermoporoelastic medium," Acta Mech. Solida Sinica, 22, No. 1, 85-92 (2009). 
9. B. Bai, "Response of saturated porous media subjected to local thermal loading on the surface of semi-space," Acta Mech. Sinica, 22, 54-61 (2006).

10. B. Bai, "Thermal response of saturated porous spherical body containing a cavity under several boundary conditions," J. Therm. Stress., 36, No. 11, 1217-1232 (2013).

11. B. Bai, "Fluctuation responses of porous media subjected to cyclic thermal loading," Comp. Geotech., 33, 396-403 (2006).

12. S. Kaushal, R. Kumar, and A. Miglani, "Responce of frequency domain in generalized thermoelasticity with two temperature," J. Eng. Phys. Thermophys., 83, No. 5, 1080-1088 (2010).

13. M. Jabbari and H. Dehbani, "An exact solution for classic coupled thermoelasticity in axisymmetric cylinder," J. Solid Mech., 2, No. 2, 129-143 (2010).

14. M. Jabbari and H. Dehbani, "An exact solution for quasi-static poro-thermoelasticity in spherical coordinate," Iran. J. Mech. Eng., 12, No. 1, 86-108 (2011).

15. I. Abbas, "Natural frequencies of a poroelastic hollow cylinder," Acta Mech., 186, No. 1, 229-237 (2006).

16. A. N. Abd-alla and I. Abbas, "A problem of generalized magnetothermo-elasticity for an infinitely long, perfectly conducting cylinder," J. Therm. Stress., 25, No. 11, 1009-1025 (2002).

17. T. He and L. Cao, "A problem of generalized magneto-thermoelastic thin slim strip subjected to a moving heat source," Math. Comp. Model., 49, 1710-1720 (2009).

18. I. Abbas, "Generalized magneto-thermoelasticity in a nonhomogeneous isotropic hollow cylinder using the finite element method," Arch. Appl. Mech., 79, 41-50 (2009).

19. I. A. Abbas, A. N. Abd-alla, and M. I. A. Othman, "Generalized magneto-thermoelasticity in a fiber-reinforced anisotropic half-space," Int. J. Thermophys., 32, 10711085 (2011).

20. I. A. Abbas, "Generalized magneto-thermoelastic interaction in a fiber-reinforced anisotropic hollow cylinder," Int. J. Thermophys., 33, 567-579 (2012).

21. I. A. Abbas and A. M. Zenkour, "LS model on electro-magneto-thermoelastic response of an infinite functionally graded cylinder," Compos. Struct., 96, 89-96 (2013).

22. I. A. Abbas and S. M. Abo-Dahab, "On the numerical solution of thermal shock problem for generalized magneto-thermoelasticity for an infinitely long annular cylinder with variable thermal conductivity," J. Comput. Theor. Nanosci., 11, No. 3, 607-618 (2014).

23. I. A. Abbas, "Fractional order GN model on thermoelastic interaction in an infinite fibre-reinforced anisotropic plate containing a circular hole," J. Comput. Theor. Nanosci., 11, No. 2, 380-384 (2014).

24. I. A. Abbas, "A GN model for thermoelastic interaction in an unbounded fiber-reinforced anisotropic medium with a circular hole," Appl. Math. Lett., 26, 232-239 (2013).

25. R. Kumar, V. Gupta, and I. A. Abbas, "Plane deformation due to thermal source in fractional order thermoelastic media," J. Comput. Theor. Nanosci., 10, No. 10, 2520-2525 (2013).

26. I. A. Abbas, R. Kumar, and V. Chawla, "Response of thermal source in a transversely isotropic thermoelastic half-space with mass diffusion by using a finite element method," Chinese Phys. B, 21, No. 8, 084601-1-084601-7 (2012). 
27. A. M. Zenkour and I. A. Abbas, "Magneto-thermoelastic response of an infinite functionally graded cylinder using the finite element model," J. Vibr. Control, 20, No. 12, 1907-1919 (2014).

28. I. A. Abbas and R. Kumar, "Interaction due to a mechanical source in transversely isotropic micropolar media," J. Vibr. Control, 20, No. 11, 1607-1621 (2014).

29. I. A. Abbas, "A GN model based upon two-temperature generalized thermoelastic theory in an unbounded medium with a spherical cavity," Appl. Math. Comput., 245, 108-115 (2014).

30. M. Jabbari and H. Dehbani, "An exact solution for classic coupled thermoporoelasticity in cylinderical coordinate," J. Solid Mech., 1, No. 4, 343-357 (2009).

31. R. Kumar and L. Rani, "Elastodynamic response of mechanical and thermal source in generalized thermoelastic half space with voids," Mech. Mechanic. Eng., 9, No. 2, 29-45 (2005).

Received 06. 11. 2014 